SPACE RESEARCH COORDINATION CENTER

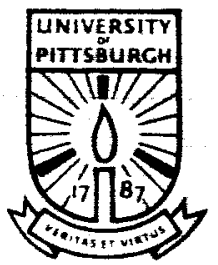

\title{
SOME CONSIDERATIONS CONCERNING RADIATION TRANSPORT IN THE OI I304 TRIPLET IN THE UPPER ATMOSPHERE
}

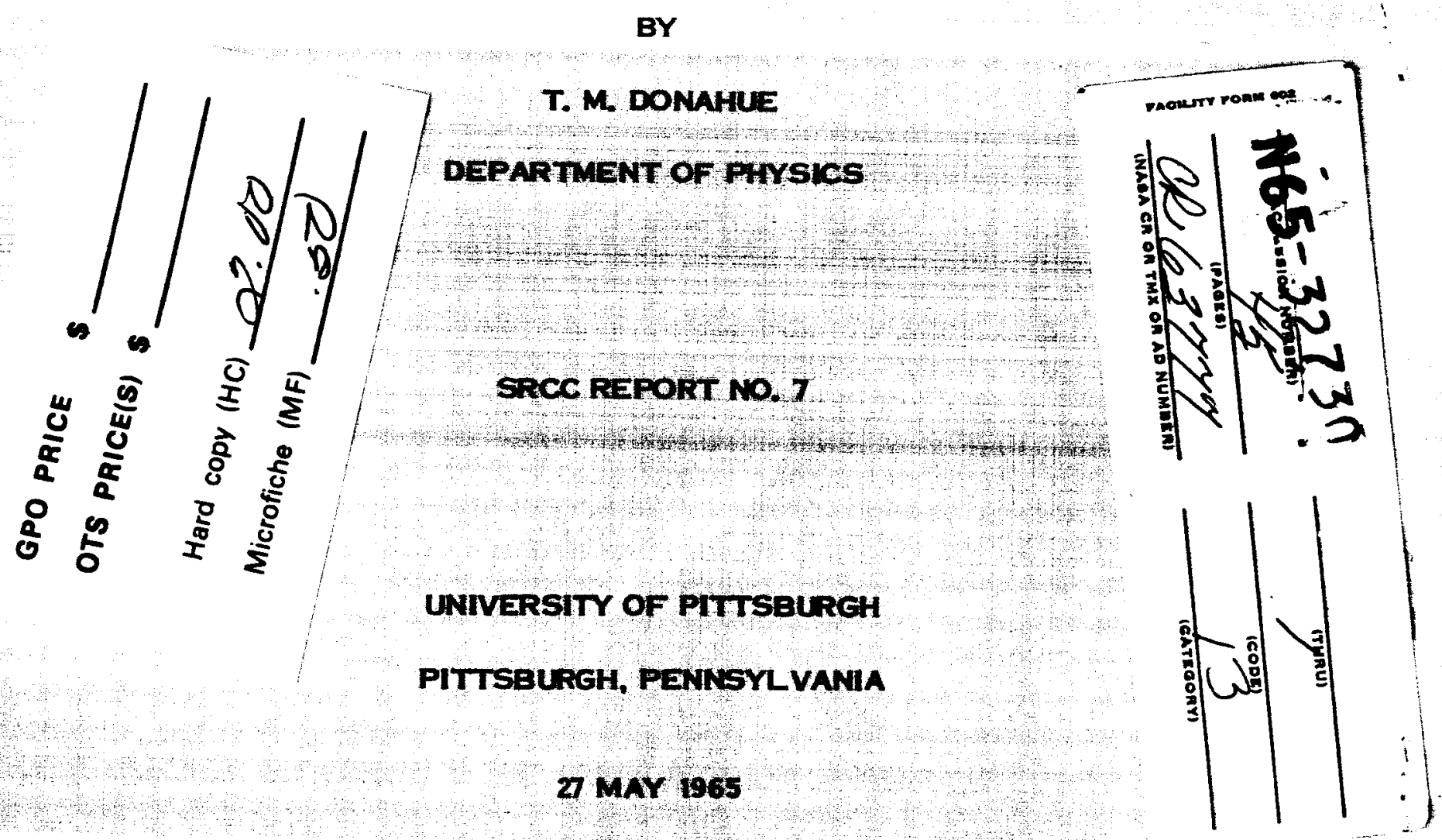


SOME CONSIDIRATIONS CONCERNING RADIATION TRANSPORT IN THE OI 1304 TRIPIET IT THE UPPER ATMOSPHERE

\author{
T. M. Donahue \\ Planetary and Space Sclence \\ University of Pittsburgh \\ Pittsburgh, Pennsylvania
}


SOME CONSIDERATIONS CONCERNING RADIATION TRANSPORT IN THE OI 1304 TRIPLET IN THE UPPER ATMOSPHERE

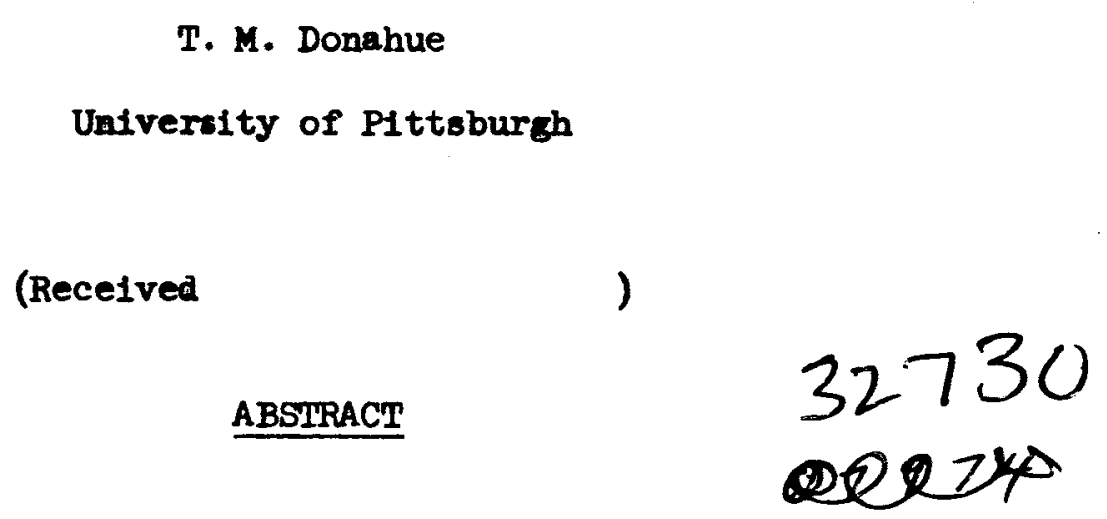

Radiation transport in an optically thick medium where the natural wings of the lines are important is discussed in connection whth the 1304 OI triplet observed in the upper atmosphere. Resonance scattering of sunlight will lead to an altitude variation of the airglow lines like the one observed but predicts only $2 \mathrm{kR}$ peak intensity at $185 \mathrm{~km}$ for solar lines $0.2 \AA$ wide. Similarly the intensity observed at $1356 \AA$ in the forbidden oxygen transition $\left({ }^{5} S-{ }^{3} P\right)$ is far too great to be resonance scattering of solar radiation. The question of the need for a source of excitation at $200 \mathrm{~km}$ remains open.

Awther 


\section{INIRODUCTION}

By this tine a nuaber of observations have been made of the 1302 $1306 \mathrm{~A}$ triplet of atonic oxycen in the dayslow of the upper atmosphere $1,2,3,4,5$. The variation of intensity with altitude has been measured from $100 \mathrm{~km}$ to above $1000 \mathrm{~km}$. A common feature of all of the profiles with one possible exception is a steep rise in the flux of downward streaming resonance photons between $100 \mathrm{~km}$ and about $190 \mathrm{~km}$ followed by a monotonic decrease to the highest altitude. The absolute intensity measurements vary quite considerably. Values have been reported at the peak ranging from 2 to $7.7 \mathrm{kR}$ even for the same solar zenith angle $\left(60^{\circ}\right)$. It is not clear whether this is a real variation or reflects the great calibration difficulties attending these measurements and the pernicious effects of small amounts of outgassing on the transmissivity near the spectrophotometers.

The radiation observed arises from the "resonance" transitions $(2 p)^{4}{ }^{3} P_{2,1,0}-(2 p)^{3} 3 s{ }^{3} S_{1}$. The observations to be discussed here were made in the direction of the zenith at a solar elongation of $60^{\circ}$. Direct sunlight was excluded from the detectors.

There are three intense solar emission lines belonging to this triplet. Their intensities at $140 \mathrm{~km}$ are $0.025 \mathrm{ergs} / \mathrm{cm}^{2} \sec \left(3_{\mathrm{P}_{0}}{ }^{-{ }^{3}} \mathrm{~s}_{1}\right)$, 0.020 ergs $/ \mathrm{cm}^{2} \mathrm{sec}\left({ }^{3} \mathrm{P}_{1}-{ }^{3} \mathrm{~S}_{1}\right)$ and 0.013 ergs $/ \mathrm{cm}^{2} \mathrm{sec}\left({ }^{3} \mathrm{P}_{2}-{ }^{3} \mathrm{~S}_{1}\right)$ and the full width of these lines it not greater than $0.2 \AA 6,7$. It is natural to suppose that the dayglow observed rises entirely or in large mearure from resonance scattering of sunlight. There is even the possibility that an important contribution to the scattered 1302-06 lines could 
be made by solar Lyman $\beta$ which is absorbed by atomic oxygen in the transition $2 p^{4}{ }^{3} P_{2}-(2 p)^{3} 3 d{ }^{3} .^{8}$ The ${ }^{3}$ state will feed the $3 s{ }^{3}$ s state efficiently by cascading.

However, Donahue and Fastie ${ }^{l}$ have shown that if the multiple resonance scattering occurs incoherently with complete frequency redistribution and the oxygen absorption lines have a pure Doppler shape no pronounced maximum should occur between $160 \mathrm{~km}$ and about $400 \mathrm{~km}$. Furthermore, the expected maximum dayglow intensity would be about $2 \mathrm{kR}$, even taking the contribution of Ly $\beta$ in the Bowen mechanism into effect. The pointed out that if the assumption of a Doppler line shape is reasonably valid it is necessary to assume the existence of an internal source of excitation near $180 \mathrm{~km}$ to account for the maximum. Subsequently excitation of atomic oxygen by energetic photo-electrons has been proposed as the mechanism for this excitation. 9,10

It is now evident that the oscillator strength of the 1302-06 transition is large. ${ }^{11,12}$ Its value appears to be at least 0.03 , and perhaps as large as $0.046^{13}$ in contrast with the value 0.0065 used in the calculation referred to. Thus scattering in the natural wings of the oxygen lines is not negligible when such great optical depths as occur in this problem are involved. 
3

RADIATIVE TRANSFER THEORY

1. The Atomic Oxygen

Following convention the frequency is measured in units of the Doppler width

$$
\begin{aligned}
x & =\left(\nu-\nu_{0}\right) / \Delta \nu_{D} \\
\Delta \nu_{D} & =\lambda_{0}^{-1} \sqrt{2 k T / m(0)}
\end{aligned}
$$

If absorption and remission always were to occur at $v_{0}$ in the rest frame of the atom the scattering cross section in the laboratory frame would have the Doppler dependence on frequency

$$
\sigma(x)=\sigma_{0} e^{-x^{2}}
$$

where

$$
\sigma_{0}=\left(\pi e^{2} / m c\right)(g f) /\left(\sqrt{\pi} \Delta \nu_{D}\right)
$$

In the atomic rest frame, however, the absorption coefficient has a dependence on frequency given by the natural resonance shape. When this frequency dependence is smeared by the atomic motions the cross section (4) becomes

$$
\begin{aligned}
\sigma(x) & =\sigma_{0} \frac{a}{\sqrt{\pi}} \int_{-\infty}^{+\infty} \frac{\exp \left(-y^{2}\right) d y}{a^{2}+(x-y)^{2}} \\
& =\sigma_{0} \exp \left(-x^{2}\right)+a H_{1}(x)+a_{2} H_{2}(x)+\cdots
\end{aligned}
$$

where

$$
H_{1}(x)=(-2 / \sqrt{\pi})[1-2 x F(x)] \cong\left(\sqrt{\pi} x^{2}\right)^{-1}
$$


for $a<0.01$ and $x \gtrsim 2$. The damping coefficient a is Given by

$$
a=\sum_{j} A_{i j} /\left(4 \pi \Delta \nu_{D}\right)
$$

where the sum is over all transitions down from the upper state. The cross section will be represented here in its approximate form

$$
\sigma(x)=\sigma_{0} \exp \left(-x^{2}\right)+\sigma_{0} a /\left(\sqrt{\pi} x^{2}\right)
$$

when $x>2$. Since, with $f=0.03$, the sum of the transition probabilities $\Sigma A 1 j$ is $3.86 \times 10^{8} \mathrm{sec}^{-1}$ and at $1000^{\circ}$ the Doppler width is $\Delta v_{D}=0.78 \times 10^{10}$ $\sec ^{-1}$, the frequency dependence of the cross section becomes

$$
\exp \left(-x^{2}\right)+ \begin{cases}3.17 \times 10^{-3} / x^{2} & T=500^{\circ} \mathrm{K} \\ 2.43 \times 10^{-3} / x^{2} & T=850^{\circ} \mathrm{K} \\ 2.24 \times 10^{-3} / x^{2} & T=1000^{\circ} \mathrm{K} .\end{cases}
$$

The value of $x$ for which the two terms in the cross section are equal is 2.86 at $1000^{\circ}, 2.80$ at $850^{\circ}$ and 2.74 at $500^{\circ}$. At $x_{c}=2.86$

$$
\sigma\left(x_{c}\right)=5,5 \times 10^{-4} \sigma_{0}
$$

The cross section at the center of the line for $T=1000^{\circ}$ is given by

$$
\sigma_{0} i=1,92 \times 10^{-12} g_{i} t_{i}
$$

Since measurement gives oscillator strengths of $0.17,0.09$ and 0.0303 the 
cross sections at the central frequency for the three lines are

$$
\begin{aligned}
& \sigma_{05}=3.2 .6 \times 10^{-13} \mathrm{~cm}^{2}\left(3 p_{2}-3 s_{1}\right) \\
& \sigma_{03}=1.73 \times 10^{-13} \mathrm{~cm}^{2}\left(3 p_{1}-3 S_{1}\right) \\
& \sigma_{01}=0.582 \times 10^{-13} \mathrm{~cm}^{2}\left(3 p_{0}-3 S_{1}\right)
\end{aligned}
$$

The density of atomic oxygen measured mass spectrometrically at various altitudes in Nay, 1963 is given in Table 1 along with the residual abundance in atoms $/ \mathrm{cm}^{2}$ above those altitudes and the gas temperature. At $1000^{\circ} \mathrm{K}$ the relative populations $\mathrm{p}_{5}, \mathrm{p}_{3}$ and $\mathrm{p}_{1}$ of the ${ }^{3} \mathrm{P}_{2},{ }^{3} \mathrm{P}_{1}$ and ${ }^{3} \mathrm{P}_{0}$ levels is $0.4,0.315$ and 0.285 .

From the cross sections (13) and the relative populations of the three levels the rertical optical thickness at the center of each line is computed as a function of altitude (Table 1 ).

$$
\tau_{i}=p_{i}^{\prime} \int n\left(z^{\prime}\right) d z^{\prime}
$$

The vertical optical thickness at frequencles $2.85,3.55$ and 4.26 Doppler units are tabulated in Table 2. The wings become important in the imprisonment problem only deep in the layer below $150 \mathrm{~km}$ for the strongest line. However, absorption of the solar lines will be much more effective than for the pure Doppler case. The question is whether the additional photons, captured in the wings, will be efficiently fed into the Doppler core where they can be trapped or rapidly leak out of the atmosphere after a few scatterings. In the latter case they will contribute little to the scattered radiation field inside the scattering medium

The accumulated number of photons scattered from the solar lines at any level well within the medium is somewhat smaller than the observed 
Table 1

\begin{tabular}{|c|c|c|c|c|c|c|}
\hline $\begin{array}{c}\text { Altitude } \\
\text { km }\end{array}$ & $\begin{array}{l}\mathrm{n}(0) \\
\mathrm{cm}^{-3}\end{array}$ & ${ }^{\mathrm{T}} \mathrm{T}$ & $\begin{array}{l}\mathrm{n}(0) \\
\mathrm{cm}^{-2}\end{array}$ & $\begin{array}{c}\tau_{5} \\
3_{P_{2}-}{ }^{3} s_{1}\end{array}$ & $\begin{array}{c}\tau_{3} \\
3_{P_{1}}-{ }^{3} s_{1}\end{array}$ & $\begin{array}{c}\tau_{1} \\
{ }^{3} P_{0}-{ }^{3} s_{1}\end{array}$ \\
\hline 110 & 1.8 & $240^{\circ}$ & $1.8(17)$ & $2.26(4)$ & $9.5(3)$ & $2.9(3)$ \\
\hline 120 & $4(10)$ & $300^{\circ}$ & $9(16)$ & $1.15(4)$ & $4.9(3)$ & $1.47(3)$ \\
\hline 140 & $1.1(10)$ & $420^{\circ}$ & $3.5(16)$ & $4.69(3)$ & $1.96(3)$ & $5.97(2)$ \\
\hline 160 & $4.5(9)$ & $600^{\circ}$ & $1.8(16)$ & $2.34(3)$ & $9.8(2)$ & $3.0(2)$ \\
\hline 180 & 2 (9) & $780^{\circ}$ & 1 (16) & $1.32(3)$ & $5.55(2)$ & $1.69(2)$ \\
\hline 200 & $1.2(9)$ & $1000^{\circ}$ & $6.4(15)$ & $8.05(2)$ & $3.38(2)$ & $1.03(2)$ \\
\hline 220 & $5(8)$ & $1000^{\circ}$ & $4 \quad(15)$ & $5.2(2)$ & $2.18(2)$ & $6.65(1)$ \\
\hline 300 & $1.7(8)$ & $1000^{\circ}$ & $7.8(14)$ & $1.0(2)$ & $3.9(1)$ & 1.2 (1) \\
\hline 350 & $7 \quad(7)$ & $1000^{\circ}$ & $2.8(14)$ & $3.6(1)$ & $1.45(1)$ & $4.4(0)$ \\
\hline 500 & $5.5(6)$ & $1000^{\circ}$ & $1.4(13)$ & $1.75(0)$ & $0.73(0)$ & $0.22(0)$ \\
\hline
\end{tabular}


downward travelling flux. Insofar as the solar lines may be treated as continua of intensity $\pi \mathrm{Fo} / \Delta v_{S}$, where $\Delta v_{S}$ is the solar line width, the number of photons scattered from one line down to an optical depth Is given by

$$
\left(\pi F_{0} / \Delta \gamma_{S}\right) \Delta \nu_{D} \int_{-\infty}^{+\infty}[1-\exp (-\tau(x) / \mu)] d x
$$

where $\mu$ is the cosine of the solar zenith angle. The integral in (15) 18 plotted in Fig. 1 as a function of optical thickness with $\mu=0.5$ for the two cross sections (3) and (9). The ratio of the atmosphertc Doppler line width to the solar line width is $1 / 22$ and the factor $\pi$ Fo $\Delta v_{D} / \Delta v_{S}$ is $3.8 \times 10^{-2} \mathrm{kR}, 5.9 \times 10^{-2} \mathrm{kR}$ and $7.3 \times 10^{-2} \mathrm{kR}$ for the three 11nes. Hence at $180 \mathrm{~km} 0.34 \mathrm{kR}, 0.43 \mathrm{kR}$ and $0.42 \mathrm{kR}$, or $1.2 \mathrm{kR}$ in $\mathrm{all}$ have been scattered from the solar lines as primary photons. Of these $0.11 \mathrm{kR}, 0.1 \mathrm{kR}$ and $0.045 \mathrm{kR}$, or about $0.3 \mathrm{kR}$ in all are scattered in the wings. The integrated primary scattering for the three lines is compared with the observed dayglow as a function of altitude in Fig. 2 .

The photons which are observed deep in the medium are photons which have been captured from the solar beam somewhere in the medium and have undergone repeated scatterings on thelr way to escape back into space or to be absorbed by other atmospheric gases lower in the atmosphere. The number of photons absorbed from the solar beam per unit optical thickess decreases steadily with optical depth. However, the number of scatterings a photon must make to escape increases in a fashion almost inverse to the capture rate so that the actual excitation rate remains high down to the level at which escape to the absorbing medium below begins to be effective. 
9

2. General Theory With Complete Redistribution

The theory of multiple scattering or imprisonment for the case of a pure Doppler line with complete frequency redistribution was developed following the methods of Holstein and Biberman in the paper by Donahue and Pastie'. It has also been discussed recently by Tomatsu. The model is a plane parallel one with the plane $\tau=\tau_{v}$ being perfectly absorbing. A continuum of intensity $\pi$ Fo/ $\Delta v$ s is incident at $\tau=0$ at an angle $\cos ^{-1} \mu$ with the vertical. The excitation rate $j(\tau)$ at $\tau$ is given by

$$
j(\tau)=j_{0}(\tau)+\int j\left(\tau^{\prime}\right) H\left(\tau, \tau^{\prime}\right) d \tau^{\prime}
$$

where $H\left(\tau, \tau^{\prime}\right) \mathrm{d} \tau^{\prime}$ is the probability that a photon emitted in the layer between $\tau^{\prime}$ and $\tau^{\prime}+d \tau^{\prime}$ is captured at the level $\tau \cdot j_{0}(\tau)$ is the rate of primary excitation, in the present case by solar photons. The transfer function $H\left(\tau, \tau^{\prime}\right)$ is related to the probability $\mathcal{E}(\Delta \tau)$ that a photon emitted at some level will cross a plane at a level $\Delta \tau$ away.

$$
H(\tau)=-\frac{\partial \varepsilon}{\partial \tau}
$$

and

$$
\varepsilon(\Delta \tau)=(1 / 4 \pi) \int_{0}^{2 \pi} d \phi \int_{0}^{\pi / 2} T(\Delta \tau \sec \theta) \sin \theta d \theta
$$

where

$$
T(\tau)=\int P(\nu) \exp (-\tau(\nu)) d \nu
$$


Is the probability that a photon emitted with the frequency spectrum $P(v)$ will travel a distance $\tau$ without being absorbed.

The probability that a photon will escape from the level $\tau$ is $E(\tau)$ where

$$
\left.E(\tau)=1-\int H\left(\tau, \tau^{\prime}\right) d \tau^{\prime}=\varepsilon(\tau)+C_{(\tau}-\tau\right)
$$

Because of this relationship the transport equation (16) may be written

$$
j^{\prime}(\tau)=\dot{j}_{0}(\tau) / E(\tau)+\int\left[j\left(\tau^{\prime}\right)-\dot{j}(\tau)\right] H\left(\tau, \tau^{\prime}\right) \phi \tau^{\prime} / E(\tau)
$$

From the computational point of view this is a very useful form of (16). $j_{0}(\tau) / E(\tau)$ is a good flrst approximation to $j(\tau)$. The integral is a correction with the help of which $j_{0}(\tau) / \mathrm{E}(\tau)$ can quickly be adjusted to yield a very close approximation to $j(\tau)$. In particular it can be noticed at once that if $j(\tau)$ has a maximum at some $\tau_{m}$ then $j\left(\tau_{m}\right)$ is less than $j_{0}(\tau m) / E\left(\tau_{m}\right)$ there and $\tau_{m}$ cannot be far removed in $\tau$ from the value of $\mathcal{V}$ at which $j_{0} / \mathrm{s}$ has its maximm.

The rate of absorption of beam photons per unit length $J_{0}(8)$ is given by

$$
\dot{j}_{0}(z)=\left(\pi F_{0} / \Delta \nu_{s}\right) \int k(\nu) \exp [-\tau(\nu) / \mu] d \nu
$$

and $k(v)$ is the absorption coefficient. From (19) this is seen to be just

$$
\dot{j}_{0}(z)=\left(\pi F_{0} / \Delta \nu_{S}\right) \sqrt{\pi} \Delta \nu_{D} k_{0} T(\tau / \mu)
$$

or

$$
f_{0}(\tau)=\left(\pi F_{0} / \Delta \nu_{S}\right) \sqrt{\pi} \Delta \nu_{D} T(\tau / \mu)
$$

per unit $\tau$. 
17

For the case of a pure Doppler line Holstein has shown that asymptotically

$$
\begin{aligned}
& T(\tau) \sim(\tau \sqrt{\pi \ln \tau})^{-1} \\
& \varepsilon(\tau) \sim(4 \tau \sqrt{\pi \ln \tau})^{-1} \\
& H(\tau) \sim[1-1 /(2 \ln \tau)]\left[4 \tau^{2} \sqrt{\pi \ln \tau}\right]^{-1}
\end{aligned}
$$

It is because $J_{0}(\tau) / E(\tau)$ varies as $T(\tau / \mu) / E(\tau L$ from $\tau=0$ almost to $\tau=\tau_{v} / 2$ and because both numerator and denominator have the same dependence on $\tau$ apart from a logarithmic factor that there is almost unfform excitation deep in the medium for a pure Doppler line case. At $\tau=\tau_{v} / 2, E(\tau)=2 E(\tau)$ and $E(\tau)$ Increases thereafter as the plane $\tau=\tau_{v}$ is approached so that $J_{0} / E$ drops to half its plateau value at $\tau_{v} / 2$ and rapidly approaches zero at $\tau_{v^{*}}$

From the excitation function $J(\tau)$ the intensity of scattered photons arriving in any direction at the level $\tau$ is given by

$$
4 \pi I(\tau)=\int j\left(\tau^{\prime}\right) T\left(\tau, \tau^{\prime}\right) d \tau^{\prime}
$$

where the integration is along the line of sight. Although the most important contributions to this integral are obtained within some 10 optical thicknesses there are non trivial numbers of photons arriving from very distant ranges of $\tau$ if $\tau$ is large because of the slow decrease with $\tau$ of $T(\tau)$. These photons are of course predominantly in the wings of the line and the spectrum of the line is broad and reversed. 
17,18

Holstein bas shown that this treatment takes into account all important effects arising from frequency redistribution on scattering for the pure Doppler case. The very strong imprisonment is a consequence of the fact that a photon absorbed at some frequency $x^{\prime}$ has equal probability of being scattered to any frequency in the range $-\left|x^{*}\right|<x<$ $|x|$ but the probability of larger shifts decreases exponentially with $\left|x^{\prime}\right|-|x|$. In the rest frame of the atom each absorption and emission is at the natural frequency. A photon far off resonance in the observer's frame may be captured only by an atom in the tail of the Maxwell distribution with a large velocity component in the direction of photon travel. Since reemission is isotropic the scattered photon as seen by the observer tends to be at a frequency closer to the natural frequency. Photons which are absorbed in the wings of the naturel line in the atom's rest frame on the other hand may be far off the center of the line in the laboratory frame without depending on capture by a high velocity atom. Thus, although redistribution strictly speaking occurs for them also, the relative probability that they are sent back into the Doppler core is reduced even though there is always a bias in that direction. The asymetry in the redistribution function decreases with 21 increasing $x$ as Unno has shown. Thus, to follow a discussion by Oster22 brook, it can be seen that the escape problem is severely modifled if a photon starts so deep in the medium that the optical depth is large compared to unity at $x_{c}$ where the natural wings begin to dominate. A photon which by chance is emitted at a frequency in the wings has a good chance after a few scatterings of being shifted back into the core. However, the spatial diffusion during the scatterings in the wings where the free paths are long can carry it close to the boundary. There it may escape 
more readily especialiy on a later excursion in frequency into the wings. The escape probability instead of varying as $(\tau \sqrt{\pi \ln \tau})^{-1}$ varies more rapidly with $\tau$ when $\mathcal{Z}$ is large enough that the wings becowe Important.

In the present problem the optical thickness for the strongest Iine becomes unity at the transition frequency between the Doppler core and the wings at about $160 \mathrm{~km}$ (Table 2). It is only below that level that the treatment outlined above begins to become questionable. In fact only at about the $130 \mathrm{~km}$ level does escape in the wings seriously begin to alter the dependence of $E(Z)$ on $Z$. Since this is about half way through the medium (measured in units of $\tau^{\prime}$ ) the pure Doppler approach can be used over most of the region of interest in the problem once jo is determined. It should be noted, however, that in a region where $\tau$ is of the order of unity at $x_{c}$ for the 1302 line it is only 0.4 for the 1304 line and 0.13 for the 1306 line. Thus a photon which happens to be absorbed well in the natural wings of the 1302 line has four chances in nine of being emitted in the wings of the other two lines where it can readily escape. On the other hand, scmewhat deeper in the medium where a photon can be absorbed in the wings of the other two lines with a reasonable probability the emitted photon has a chance of $5 / 9$ to be emitted in the 1302 line where it has a small chance of going far without being reabsorbed. Thus although the wings will have little influence on the imprisonment problem once a photon is absorbed in the Doppler core the principal question is to determine at what rate photons captured from the solar beam in the natural wings are fed into the core. There is a supply of photons here which becomes more and more important relative to the direct absorption in the core as the beam progresses into the medium. If a 
Table 2

\begin{tabular}{|c|c|c|c|}
\hline \multirow{2}{*}{$\begin{array}{c}\text { Altitude } \\
\mathrm{km} \\
\end{array}$} & \multicolumn{3}{|c|}{ At $x=2.86$} \\
\hline & $5^{(2.86)}$ & $\tau_{3}(2.86$ & $\tau_{1}(2.86)$ \\
\hline 110 & 12.4 & 5.33 & 1.60 \\
\hline 120 & 6.35 & 2.70 & 0.81 \\
\hline 140 & 2.58 & 1.08 & 0.33 \\
\hline 160 & 1.29 & .54 & 0.17 \\
\hline 180 & 0.73 & .31 & 0.09 \\
\hline 200 & 0.44 & .19 & 0.06 \\
\hline 220 & 0.28 & .12 & 0.04 \\
\hline & & $x=3$ & \\
\hline $\begin{array}{c}\text { Altitude } \\
\text { km }\end{array}$ & $\tau_{5}(3.55)$ & $x_{3}($ & $\tau_{1}(3.55)$ \\
\hline 110 & 8.1 & 3.3 & 1.0 \\
\hline 120 & 4.1 & 1.7 & .52 \\
\hline 140 & 1.7 & .69 & .21 \\
\hline 160 & .81 & .34 & .10 \\
\hline 180 & .47 & .20 & .06 \\
\hline 200 & .28 & .12 & .04 \\
\hline 220 & .18 & .07 & .02 \\
\hline
\end{tabular}


Table 2 - cont'd.

\begin{tabular}{|c|c|c|c|}
\hline \multirow{2}{*}{\begin{tabular}{c} 
Altitude \\
\cline { 2 - 4 }
\end{tabular}} & $\tau_{5}(4.26)$ & $\tau_{3}(4.26)$ & $\tau_{1}(4.26)$ \\
\hline 110 & 5.6 & 2.4 & 0.7 \\
120 & 2.8 & 1.2 & 0.23 \\
140 & 1.2 & .31 & 0.09 \\
160 & .58 & .15 & 0.05 \\
180 & .32 & .09 & 0.03 \\
200 & .2 & .05 & 0.02 \\
220 & .12 & .03 & 0.01 \\
\hline
\end{tabular}


sizeable fraction of these photons can be shifted into the core then there is a possibility of producing an increasing imprisoned excitation with increasing $\tau$ and the observed increase in brightness down to $180 \mathrm{~km}$ may be realized.

In this case, however, a difficulty will be to account for the decrease in intensity from $180 \mathrm{~km}$ down to $110 \mathrm{~km}$. The $180 \mathrm{~km}$ level is only about $1 / 20$ of the way to $110 \mathrm{~km}$ in units of optical thickness. It is only at $110 \mathrm{~km}$ that the number of scatterings suffered by an imprisoned photon before escape becomes so large that its total path length in the atmosphere becomes comparable to the mean free path for absorption in $\mathrm{O}_{2}$. The cross section for absorption of a 1300 photon by $\mathrm{O}_{2}$ is of the order of $10^{-18} \mathrm{~cm}^{2}$. The absorption coefficient $\sigma_{\mathrm{A}^{n}} \mathrm{n}\left(\mathrm{O}_{2}\right)$ is tabulated at various altitudes in Table 3. It becomes about $2 \times 10^{-7} \mathrm{~cm}^{-1}$ at $110 \mathrm{~km}$. Taking the average cross section for resonance scattering to be $10^{-13} \mathrm{~cm}^{2}$ the scattering coefficient $\sigma_{R} n(0)$ can be calculated. This is $1.5 \times 10^{-1}$ at $110 \mathrm{~km} .^{*}$ Thus a photon can on the average suffer $\sigma_{R} n(0) / \sigma_{A} n\left(O_{2}\right)$ scatterings before absorption. At $110 \mathrm{~km}$ (Table 3) this number is $8.5 \times 10^{4}$. On the other hand, the optical thickness to infinity for the strongest line there is about $2.3 \times 10^{4}$ and $4 \tau \sqrt{\pi \ln \tau}$ which is $1 / E(\tau)$ and hence measures the number of scatterings before escape is $5 \times 10^{5}$. At $120 \mathrm{~km}$ the same two quantities are $1.3 \times 10^{5}$ and $2.5 \times 10^{5}$. This is for the strongest line. Because the photon spends half of its Iife in the other two lines and in fact the imprisonment is weakened by escape in the wings absorption begins to be effective somewhere between 110 and $120 \mathrm{~km}$. In this paper the absorbing lower boundary has been put at $110 \mathrm{~km}$.

\footnotetext{
* It is interesting that the photon mean free path is small compared to the dimensions of a rociket below $140 \mathrm{~km}$. This should affect the measured intensities in that the rocket creates a sheath of low excitation about itself.
} 
Table 3

\begin{tabular}{|c|c|c|c|c|}
\hline $\begin{array}{c}\text { Altitude } \\
\mathrm{km}\end{array}$ & $\begin{array}{c}\sigma_{A} n\left(O_{2}\right)=K\left(0_{2}\right) \\
\mathrm{cm}^{-1}\end{array}$ & $\begin{array}{l}\mathrm{S}_{0}(0) \\
{ }^{3} \mathrm{P}_{2}-{ }^{-3} \mathrm{~S}_{1}\end{array}$ & $\mathrm{~K}(\mathrm{O}) / \mathrm{K}\left(\mathrm{O}_{2}\right)$ & 1/E( .) \\
\hline 110 & $1.75(-7)$ & $1.5(-2)$ & $8.5(4)$ & $5(5)$ \\
\hline 120 & $3.8(-8)$ & $5(-3)$ & $1.3(5)$ & $2.5(5)$ \\
\hline 140 & $4.9 \quad(-9)$ & $1.4(-3)$ & $2.9(5)$ & $1.1(5)$ \\
\hline 160 & $9 \quad(-10)$ & $5.4(-4)$ & $6 \quad(5)$ & $5.1(4)$ \\
\hline 180 & $2.3(-10)$ & $2.8(-4)$ & $1.2(6)$ & $2.5(4)$ \\
\hline 200 & 7.5 (-11) & $1.2(-4)$ & $1.6(6)$ & $1.1(4)$ \\
\hline
\end{tabular}


16

To calculate $j_{0}$ it is necessary to evaluate the function

$$
j_{0}(\tau)=\left(\pi F_{0} / \Delta \nu_{s}\right) \Delta \nu_{0} \int_{-\infty}^{+\infty}\left(k(x) / \kappa_{0}\right) \exp [-\tau(x) / \mu] d x
$$

The integrand on the right hand side of (29) for small values of $\tau$ is dominated by the Doppler core and has the form

$$
e^{-x^{2}} \exp \left[-(\tau / \mu) e^{-x^{2}}\right]
$$

But for sufficiently large $\tau$ the wings predominate and the integrand may be approximated by

$$
\left(a / \sqrt{\pi} x^{2}\right) \exp \left[-(\tau a) /\left(\sqrt{\pi} \mu x^{2}\right)\right] .
$$

Hence for $\tau$ small enough that $\tau\left(x_{c}\right)<1$

$$
\begin{aligned}
j o(\tau) & \cong\left(\pi F_{0} / \Delta \nu_{S}\right) \Delta \nu_{D} \sqrt{\pi} \quad \frac{1}{\sqrt{\pi}} \int_{-\infty}^{+\infty} e^{-x^{2}} \exp \left[-(\tau / \mu) e^{-x^{2}}\right] d x \\
& =\left(\pi F_{0} / \Delta \nu_{S}\right) \sqrt{\pi} \Delta \nu_{D} T(\tau / \mu) \\
& \sim\left(\pi F_{0} / \Delta \nu_{S}\right) \sqrt{\pi} \Delta \nu_{D}[(\tau / \mu) \sqrt{\pi \ln (\tau \mid \mu)}]^{-1}
\end{aligned}
$$

and this gives the rate per unit $\tau$ that solar photons are absorbed. On the other hand for $\tau$ so large that $\tau\left(x_{c}\right)>>1$ - or in general for the rate at which photons are absorbed in the natural wings it is necessary to evaluate

$$
\left(\pi F_{0} / \Delta v_{s}\right) \Delta \nu_{D} \frac{a}{\sqrt{\pi}} \int_{x_{c}}^{\infty} \frac{1}{x^{2}} \exp \left[-(a \tau) /\left(\sqrt{\pi} x^{2} \mu\right)\right] d x, \text { (33) }
$$


This is practically

$$
\left(\pi F_{0} / \Delta \nu_{s}\right) \sqrt{\pi} \Delta \nu_{D} \sqrt{(a \mu)(\sqrt{\pi} \tau)}
$$

The functions $[(\tau / \mu) \sqrt{\pi \ln (\tau / \mu)}]^{-1}$ and $\left(\mu a / \sqrt{\pi} \tau^{\prime}\right)^{1 / 2}$ for the value $a / \sqrt{\pi}$ relevant to this problem are plotted in Fig. 3. For values of $\tau / \mu$ less than 100 the first of these functions accurately gives the rate of absorption and for values of $\tau / \mu$ larger than about 1000 the second is a good approximation the rate of absorption in the core being small compared to that in the wings.

However, for our purposes only the photons absorbed in the wings not too far in frequency from $x_{c}$ have a reasonable chance of shifting into the core before they scatter out of the medium. Unno has shown that the frequency redistribution function for scattering in the wings is asymetrical. There is always a larger probability that the photon will have its frequency shifted back toward the center of the line than that it will be shifted toward the wings. This bias decreases, however, with increasing frequency defect. Osterbrook has demonstrated that it is possible to approximate the exact diffusion history by requiring the photon to suffer a discrete frequency shift of $1 / \sqrt{2}$ upon scattering at $x^{\prime}>x_{c}$. The probability of a shift by $1 / \sqrt{2}$ to a frequency $x$ closer to the center of the line is $\left(1+\sqrt{2} /\left|x^{\prime}\right|\right) / 2$ wille the probability of a shift by $1 / \sqrt{2}$ away from the center is $\left(1-\sqrt{2}\left|x^{\prime}\right|\right) / 2$. Thus, for photons in the wings a dffusion treatment may be used in frequency and in space but, except far in the wings, the bias tending to send the photon back to $|x|=x_{c}$ must be taken into account. In our problem $x_{c}$ is $2.85, x_{c}+1 / \sqrt{2}$ is 3.56 and the next jump is to 4.26 Doppler units. At an $x$ of 3.56 the probability of scattering back into the core is 0.6 . At 4.26 it is 0.58 . 
This scheme permits an evaluation of the rate at which solar photons in the wings are shifted into the Doppler core and become trapped. Thus in the 1302 solar Ine about 60 per cent of the photons absorbed per unit optical thickness between $x=2.85$ and $x=3.56$ are assumed to find themselves after scattering in the core of one of the three lines, $5 / 9$ in the 1302 line, $1 / 3$ in the 1304 and $1 / 9$ in the 1306. In the case of the latter two lines the rete of injection per unit optical thickness for each of them must be obtained. This entalls transforming the rates in terms of 1302 optical thickness by factors of $1 / 0.42$ and $1 / 0.128$, the ratios of the cross sections times the effective abundance. Similarly in the other two solar lines photons at frequencies 2.85 to 3.56 Doppler units from the Iine center will feed the Doppler mechanism in all three airglow lines. Because of the swall opacities in the wings and the presence of probability factors of the order of $0.4 \times 0.6 \times 0.6$ compared to 0.6 it is safe except deep in the medium to neglect those photons which in their first scattering shift away from the core. These photons which scatter more than once in the wings before reaching the core undergo their first scattering far away from the level at which they finally reach the core. In calculating $j_{0}(\tau)$ at large $\tau$ it is necessary in principle to consider photons which are scattered first at small values of $\tau$ and continue to travel dowmard in the wings until they finally shift into the core. This is because the rate of primary scattering is so much larger at small $\tau$ than it is at large $\tau$ (Fig. 3). However, the fact that only half of the photons scattered at any level continue dowmard and the fact that the chance of a shift to a frequency further in the wings is not negligible cut down seriously on the multiple scattering contribution by wing photons to $j_{0}(\tau)$. In addition there is another effect associated with the decrease in temperature between $200 \mathrm{~km}$ and $100 \mathrm{~km}$ to be discussed later which minimizes this 
contribution. On the other band there is a non negligible contribution to $j_{0}(\tau)$ from photons scattered beyond $|x|=3.56$ below $140 \mathrm{~km}$ which shift into the range of $x$ between 2.85 and 3.56 , travel upward as 1302 photons, and scatter again neer $200 \mathrm{~km}$ into the core. From Table 2 which gives the optical thickness in the three lines at $x$ values of $2.85,3.56$ and 4.26 it can be seen that this effect is negligible in the 1306 line and effective in the 1304 line only below $150 \mathrm{~km}$ because the mean free path in $\tau$ is $1 / \sqrt{2}$ which is greater than the aistance to the top of the atmosphere.

It is necessary to know therefore the rate of absorption of solar photons in the wings for the range $2.85 \leqslant x \leqslant 3.56$ and also for the range $3.56 \leqslant x \leqslant 4.26$. These are given by $j_{0}^{\prime \prime}(\tau)$ and $f_{0}^{\prime \prime}(\tau)$ where

$j_{0}^{\prime \prime}(\tau)=\left(\pi F_{0} / \Delta \nu_{s}\right) \Delta \nu_{D}(2 a / \sqrt{\pi}) \int_{2,85}^{3,56} x^{-2} \exp \left[-(a \tau) /\left(\sqrt{\pi} \mu x^{2}\right)\right] d x$

or

$j_{0}^{\prime \prime}(\tau)=\left(\pi F_{0} / \Delta \gamma_{S}\right) \sqrt{\pi} \Delta \gamma_{D} \frac{1}{\sqrt{\pi}} \sqrt{\frac{a \mu}{\sqrt{\pi} \tau}}\left\{\Phi\left(\sqrt{\frac{a \tau}{\sqrt{\pi} \mu}} \frac{1}{3.56}\right)-\Phi\left(\sqrt{\frac{a \tau}{\sqrt{\pi \mu}} 1.25}\right\}(36)\right.$

where $\Phi(y)$ is the error integraly

$$
\Phi(y)=2 \int_{0} e^{-t^{2}} d t
$$

The factor

$$
F(\tau / \mu)=\frac{1}{\sqrt{\pi}} \sqrt{\frac{a \mu}{\sqrt{\pi \tau}}}\left\{\Phi\left(\sqrt{\frac{a \tau}{\sqrt{\pi} \mu}} \frac{1}{3.56}\right)-\Phi\left(\sqrt{\frac{a \tau}{\sqrt{\pi} \mu}} \frac{1}{2.85}\right)\right\}
$$


along with the similar quantity for the range $3.56-4.26$ are plotted in Fig. 3 for comparison with $[(\tau / \mu) \sqrt{\pi \ln (\tau / \mu)}]^{-1}$ which measures in a sintlar way the direct absorption per untt $\tau$ in the Doppler 11ne. Above a $\tau / \mu$ of about 2500 (180 $\mathrm{km}$ for 1302) the two are comparable in size.

The rate of excitation of the ${ }^{3} \mathrm{~S}_{1}$ level by solar photons close enough to resonance that the reemitted photons will be in the Doppler core must now be computed. Expressed in units of optical thickness for the 1302 line this is made up of contributions from the solar 1302, 1304 and 1306 Iines. At a given altitude it consists of

$$
\left(\pi F_{0}(1302) / \Delta v_{s}\right) \sqrt{T} \Delta v_{D}
$$

times the contribution of

$$
\text { 1302: } \begin{aligned}
& {\left[\left(\tau_{5} / \mu\right) \sqrt{\pi \ln \left(\tau_{5} / \mu\right)}\right]^{-1} } \\
& +0,65 F_{1}\left(\tau_{5} / \mu\right)
\end{aligned}
$$

and that of

$$
\text { 1304: } \frac{\pi F_{0}(1304)}{\pi F_{0}(1302)} \times 0.42\left\{\left[\left(\tau_{3} \mid \mu\right) \sqrt{\pi \ln \left(\tau_{3} / \mu\right.}\right]^{-1}+0,65 F_{1}\left(\tau_{3} \mid \mu\right)\right\}
$$

plus that of

$$
1306: \frac{\pi F_{0}(1306)}{\pi F_{0}(1302)} \times 0.128\left\{\left[\left(\tau_{1} / \mu\right) \sqrt{\pi \ln \left(\tau_{1} / \mu\right)}\right]^{-1}+0.65 F_{1}\left(\tau_{1} / \mu\right)\right\}_{(39)}
$$

At 180 the ratio of direct excitation in the core to the contribution from the wings by the 1302 solar line is $0.8 / 0.33$, by the 1304 line, 
$1.30 / 0.33$ and by the 1306 line, 1.75/0.2. The ratio of the excitation rate derived in this way to direct excitation in the core 18 1.22. In contrast the ratio at $220 \mathrm{~km}$ is 1.13 .

At $160 \mathrm{~km}$ excitation in the core via the vings relative to that In the core directly would be $5.7 / 19.6$ and the ratio of total excitation to direct core excitation would be 1.29 if $\Delta v_{D}$ were independent of altitude. However, below $200 \mathrm{~km}$ the temperature beglns to decrease rapidly from $1000^{\circ}$ to about $500^{\circ}$ at $500 \mathrm{~km}$ and $300^{\circ}$ at $120 \mathrm{~km}$. This causes $\Delta v_{D}$ to decrease considerably. $x_{c}$ is reduced to 2.74 at $500^{\circ}$. The supply of solar photons for direct excltation in the core as well as for absorption in the nearby rings (which now reach only to $|x|=3.45$ ) is drastically reduced. The eligible photons have been absorbed already by atoms higher up in the atmosphere. This is a very serlous effect and excitation by direct absorption in the vings decreases sharply below the thermopause. Furthermore, the frequencies in the vings at which solar photons are being actively absorbed are now considerably farther removed from the boundary between core and wings so that more scatterings are required to shift their frequencies into the core.

In the Imprisomment problem it is the ratio of $\mathrm{J}_{0}$ to the escape probability $\mathrm{E}$ wich determines the first approximation to the excitation function. It is true that for a pure Doppler line the escape probability In the upward direction is reduced in the same way as the primary excitation function $j_{0}$ if the temperature decreases downard. This is not the case where the wings have also been effective in removing photons which could get into the Doppler core. Furthermore the escape probability dowmard from a certain level - say near $150 \mathrm{~km}$ is vastly increased because 
of the shrinking Doppler width below that level which mokes the gas transparent in the wings of the Doppler line. Hence the direct contribution to $j_{0}$ is taken to vanish at $140 \mathrm{~km}$ and decrease monotonically between $180 \mathrm{~km}$ and $140 \mathrm{~km}$. Furtherwore the region in the wings from which photons can reach the core is shifted from $2.85<|x|<3.56$ to $2.74<|x|<3.45$ (F1g. 3) at $150 \mathrm{~km}$ and continues to shift as the altitude decreases. At $140 \mathrm{~km}$ the ratio of the excitation rate by frequency shift in the wings to the pure Doppler, constant temperature, excitation rate is $2.4 / 10$.

The ratio of the excitation rate obtained in this way to that which would occur for a pure Doppler line is shown as a function of $\tau$ in Fig. 4. There remain to be counted those photons which are extracted from the solar beam at frequencies between 0.707 and 1.414 Doppler untts from $x_{c}$, suffering a shift of 0.707 units toward $x_{c}$ upon scattering. After travelling through an optical thickness of 0.707 at their frequency they will be scattered again and some of them will find themselves afterward in the Doppler core. For example, at $205 \mathrm{~km}$ the absorption factor (38) for $x$ between 3.55 and 4.26 is $1.1 \times 10^{-4}$ in the 1302 line, $1.9 x$ $10^{-4}$ in the 1304 and $2.2 \times 10^{-4}$ in the 1304 line per unit optical thickess in each of these lines (FIg. 3). A fraction 5/9 of these 1302 photons are shifted to an $x$ less than 3.56 with a prollability of 0.6 and one balf travel dowmrard to $155 \mathrm{~km}$, (a distance $\Delta \tau(1302)$ of 0.707 at $x=3.55$ according to Table 2). The 1304 contribution is similarly determined except that it is weighted by 1.55 to take into account the higher solar intensity and also by 0.42 to put it in terms of unit optical thickness at 1302 . The same velghting factors for 1306 are 1.9 and 0.128 . In all 
$1.45 \times 10^{-5}$ is the sum of the three absorption flectors representing photons which arrive at $155 \mathrm{~km}$ in the 1302 line where $60 \%$ then have frequencies shifted into the core. This contribution of $2.76 \times 10^{-5}$ compares with the single scattered contribution of $2.4 \times 10^{-5}$ and the sum 1 s 0.52 times what would have been the Doppler line contribution alone in an isothermal layer.

\section{Final Evaluation of the Source Function}

The initial rate of excitation of the ${ }^{3} \mathrm{~s}$ level in the Doppler core, $j_{0}$, relative to $J_{0}$ for the pure Doppler shape model including the multiple scattering contributions is shown in Fig. 4.

For the pure Doppler line case $j_{0}(\tau) / E(\tau)$ is proportional to $T(\tau / \mu) / z(\tau)$. With $\mu=0.5$ this is asyntotically $4 \tau \sqrt{\ln \tau}\left(\frac{1}{2} \sqrt{\ln 2 \tau}\right)$ and the relative rates fust calculated may be converted to an absolute scale by multiplying them by

$$
\left[\frac{\pi F_{0}(1302)}{\Delta \gamma_{S}} \sqrt{\pi} \Delta \gamma_{D}\right] 2 \sqrt{\frac{\ln \tau}{\ln 2 \tau}}
$$

The result, $j_{0}(\tau) / E(\tau)$ is show in Fig. 5. From $j_{0}$ it is next necessary to obtain the steady state excitation rate $j(\mathcal{I})$ by solving the transfer equation (16) in the form

$$
j(\tau)=j_{0}(\tau)+\int \sum j_{i}\left(\tau^{\prime}\right) H\left(\tau, \tau^{\prime}\right) d \tau^{\prime}
$$

where the sum is over the three fine structure components and

$$
\begin{aligned}
& j(\tau)=\sum_{1} j_{i}(\tau) \\
& f_{5}(\tau)=\frac{5}{9} j(\tau) \text { etc. }
\end{aligned}
$$


24

Using the relationships

$$
E_{i}=1-\int H_{i}\left(\tau^{\prime}\right) d \tau^{\prime}
$$

the transfer equation (40) may be written

$$
\begin{aligned}
j(\tau)=j_{0}(\tau) & +j_{5}(\tau)-j_{5}(\tau) E_{5}(\tau)-\int j_{5}(\tau) H_{5}\left(\tau, \tau^{\prime}\right) d \tau^{\prime} \\
& +j_{3}(\tau)-j_{3}(\tau) E_{3}(\tau)-\int j_{3}(\tau) H_{3}\left(\tau, \tau^{\prime}\right) d \tau^{\prime} \\
& +j_{1}(\tau)-j_{1}(\tau) E_{1}(\tau)-\int j_{1}(\tau) H_{1}\left(\tau, \tau^{\prime}\right) d \tau^{\prime} \\
& +\int \sum_{i} j_{i}\left(\tau^{\prime}\right) H\left(\tau, \tau^{\prime}\right) d \tau^{\prime}
\end{aligned}
$$

(42)

$$
\begin{aligned}
& \text { or } j_{s}(\tau) E_{5}(\tau) \\
&+j_{3}(\tau) E_{3}(\tau)+j_{1}(\tau) E_{1}(\tau)=j_{0}(\tau) \\
&+\int \sum_{i}\left[j_{i}\left(\tau^{\prime}\right)-j_{i}(\tau)\right] H_{i}\left(\tau, \tau^{\prime}\right) d \tau^{\prime}
\end{aligned}
$$

which may also be written

$$
\begin{aligned}
& j(\tau)\left[\frac{5}{9} E_{5}(\tau)+\frac{3}{9} E_{3}(\tau)+\frac{1}{9} E_{1}(\tau)\right]=j_{0}(\tau) \\
& +\int\left[j^{\prime}\left(\tau^{\prime}\right)-j(\tau)\right]\left[\frac{5}{9} B_{5}\left(\tau, \tau^{\prime}\right)+\frac{3}{9} H_{3}\left(\tau, \tau^{\prime}\right)+\frac{1}{9} H_{1}\left(\tau, \tau^{\prime}\right)\right] d \tau^{\prime}
\end{aligned}
$$

(43) 
25

Finally with

$$
E(\tau)=\frac{5}{9} E_{5}(\tau)+\frac{3}{9} E_{3}(\tau)+\frac{1}{9} E_{1}(\tau)
$$

and

$$
H\left(\tau, \tau^{\prime}\right)=\frac{5}{9} H_{5}^{\prime}\left(\tau, \tau^{\prime}\right)+\frac{3}{9} H_{3}\left(\tau, \tau^{\prime}\right)+\frac{1}{9} H_{1}\left(\tau, \tau^{\prime}\right)
$$

or

$$
E(\tau)=1-\int H\left(\tau, \tau^{\prime}\right) d \tau^{\prime}
$$

the transport equation is found to be identical in form with (al) which was developed for a two level model. By means of this integral equation (21) the source function $g_{0}(\tau)$ may easily be transformed into the excitation function $g(\tau)$. In Fig. $5 g(\tau)$ is shown as the rate of excitation of the ${ }^{3}$ s state per unit optical thickness in the $1302\left({ }^{3} P_{2}-{ }^{3} S_{1}\right)$ transition.

4. Intensities

From $j(\tau)$ the intensities may then be obtained. Since $g(\tau)$ is already expressed as a function of $\tau_{5}$ for the 1302 line the 1302 intensity at $\tau$ is given by

$$
4 \pi I_{5}(\tau)=\frac{5}{9} \int j\left(\tau^{\prime}\right) T\left(\tau, \tau^{\prime}\right) d \tau^{\prime} .
$$

Since to obtain the other components $j\left(\tau^{\prime}\right)$ should be expressed in units of the appropriate optical thickness it is necessary to make use of the fact that

$$
\begin{aligned}
& j\left(\tau_{3}\right)=(1 / 0.42) j\left(\tau_{5}\right) \\
& j\left(\tau_{1}\right)=(1 / 0.128) j\left(\tau_{5}\right)
\end{aligned}
$$


The number of excited atoms per unit optical depth in the reaker lines is much larger than the number per unit $\tau$ in 1302 . Then

$$
\begin{aligned}
& 4 \pi I_{3}(\tau)=\frac{1}{3} \int j\left(\tau_{3}^{\prime}\right) T\left(\tau, \tau^{\prime}\right) d \tau^{\prime} \\
& 4 \pi I_{1}(\tau)=\frac{1}{9} \int j\left(\tau^{\prime}\right) T\left(\tau, \tau^{\prime}\right) d \tau^{\prime}
\end{aligned}
$$

where the excitation functions in each integral are those appropriate to each transition. If the number of atoms in each absorbing level ${ }^{3} P_{2}$, $3_{\mathrm{P}_{1}}, 3_{\mathrm{P}_{0}}$ were equal the intensities of the three components would be nearly equal deep in the medium since the ratios of the optical thicknesses would be just $5 / 3 / 1$.

Important contributions to these integrals come fram very large distances. For example at $180 \mathrm{~km}$ they give $0.50 \mathrm{kR}, 0.63 \mathrm{kR}$ and $0.62 \mathrm{kR}$ for the 1302,1304 and 1306 Ines respectively. Of these intensities

$0.40 \mathrm{kR}$ of 1302 radiation comes from $1320>\tau>1220$ or $180-184 \mathrm{~km}$ $0.09 \mathrm{kR} \quad 1220>\tau>320$ or $184-240 \mathrm{~km}$ $0.15 \mathrm{kR} \quad 320>\tau>0$ or $240-\infty$

$0.57 \mathrm{kR}$ of 1304 radiation comes from $555>\tau>455$ in $\tau$ or $180-188 \mathrm{~km}$ $0.07 \mathrm{kR} \quad 455>\tau>0 \quad 188-\infty$

$0.45 \mathrm{kR}$ of 1306 radiation comes from $170>\tau>160$ $180-183 \mathrm{~km}$ $0.17 \mathrm{kR}$ $160>\tau>70$ $183-218 \mathrm{~km}$

$0.015 \mathrm{kR}$ $70>\tau>0$ 218-00 
The calculated Intensities for the three components are given in Table 4. To these mast be added a not completely insignificant contribution in the vings. This scattering was discussed in section 1 . Its magnitude is indicated also in Table 4 and the sum of the imprisoned or multiply scattered component and the (practically) single scattered component in the vings gives the flnal overhead intensity. The maximum occurs at $180 \mathrm{~km}$ and is about $2 \mathrm{kR}$. There is a monotonic decrease above and below that altitude. The result is compared with the observations in Fig. 6. Apart from the fact that the measured intensities have varied by a factor of 4 the agreement is excellent. Since there are large uncertainties In the absolute values for the measured dayglow intensities and the measured solar intensities this comparison cannot yet be considered definttive. The solar line width used is a lower limit determined by the slit width of the IRL spectrometer. Obviously if the the doif line are one fourth the slit width agreement with the May 1963 flight of fast1e would be perfect.

As Donahue and Fastie have shown a weak source of exc1tation within the optically thick part of the oxygen atmosphere (below about $300 \mathrm{~km}$ ) would produce an intense glow if its contribution to $j_{0}$ were large compared to the solar scattering. They argued for such a source not only on the Brounds that the airglow Intensity was larger than expected but also because of the existence of the sharp maximum. This second argument is no longer compelling and the first one never was. Nevertheless the present comparison of theory and observation cannot be taken as demonstrating the absence of such a source. It no longer is clearly necessary but it atill might be present. Until precise information is on hand about the intensity 
Table 4

\begin{tabular}{|c|c|c|c|c|c|c|c|c|c|}
\hline Altitude & $\frac{101+101}{1302}$ & $\frac{e \text { seat }}{1304}$ & $\frac{\text { ring }}{1306}$ & $\begin{array}{l}\text { Sing } \\
1302\end{array}$ & $\begin{array}{l}\text { Scatt } \\
\frac{(\text { Winge) }}{1304}\end{array}$ & $\begin{array}{l}1306 \\
1306\end{array}$ & 1302 & $\frac{\text { Total }}{1304}$ & 1306 \\
\hline 500 & 0.17 & -- & -- & - & -- & $-\infty$ & 0.17 & & \\
\hline 380 & 0.27 & 0.29 & 0.20 & $--x$ & -- & $\therefore$ & 0.27 & 0.29 & 0.20 \\
\hline 250 & 0.37 & 0.38 & 0.44 & 0.03 & 0.005 & $-\infty$ & 0.40 & 0.39 & 0.44 \\
\hline 180 & 0.49 & 0.63 & 0.62 & 0.1 & 0.1 & 0.05 & 0.59 & 0.73 & 0.67 \\
\hline 160 & 0.40 & 0.55 & 0.53 & 0.15 & 0.15 & 0.09 & 0.55 & 0.70 & 0.62 \\
\hline 140 & 0.29 & 0.35 & 0.38 & 0.2 & 0.2 & 0.15 & 0.49 & 0.55 & 0.53 \\
\hline 120 & 0.10 & 0.15 & 0.16 & 0.26 & 0.30 & 0.25 & 0.36 & 0.45 & 0.41 \\
\hline
\end{tabular}


and shape of the solar lines at the time of an airglow observations and the precision of the airglow intensity measurements is increased to the degree that experiment and theory may be compared with confidence for factors of 1.25 in agreement the question of a source remins open. Kaplan, Katyushina and Kurt have measured the intensity of the 1302-06 airglow lines up to $500 \mathrm{~km}$ for very low sun angles, $5^{\circ}, 3^{\circ}$ and $-17^{\circ}$. Their results for the flight of October $25,1963\left(+3^{\circ}\right)$ fit the expected pattern quite well (Fig. 6). Because of the long slant path the absorption in the wings becomes effective about one third as far from $\tau=0$ as in the case discussed in detail in this paper. This puts their maximum at about $250 \mathrm{~km}$ and makes it rather broad. On the other hand the results of their flight on June 6, 1963 for $+5^{\circ}$ are altogether different and very perplexing. They find a sharp rise in intensity (at least on descent) between 100 and $120 \mathrm{~km}$ to a maximum at about $180 \mathrm{~km}$ then a very slow decrease to a plateau from 340 to $500 \mathrm{~km}$ on which the intensity is $2 / 3$ its peak value.

\section{THE 1356 IINE} The forbidden lines at $1356 \AA\left({ }^{5} 3_{2}-{ }^{3} P_{2,1}\right)$ observed in the airglow by Fastie may provide evidence on this question. Application of the technique of radiative transfer calculation outlined in this paper to the case of a medium of intermediate opacity predicts intensity as a function of $\tau$ shown in Fig. 7. Details concerning these calculations will be published separately. The shape of the intensity versus altitude curve observed fits best to the theoretical model in which the absorbing base is put effectively at $122 \mathrm{~km}$ and the opacity at the center of the 
line taken to be about 4 (FIg. 6). H1gher opacity produces too sharp a decrease below the peak and lower opacity will not permit a maximum above the altitude at which true absorption becomes serious.

According to the IRL observations the soler nux ( $\left.T_{F_{0}}\right) v$ at $1356 \AA$ is only about $4 \times 10^{8}$ photons $/ \mathrm{cm}^{2} \sec \AA$ or $2.4 \times 10^{4}$ photons $/ \mathrm{cm}^{2}$ per untt frequency. Hence the factor

$$
\left(\pi F_{0}\right)_{\nu} \sqrt{\pi} \Delta v_{0}
$$

is only $3.3 \times 10^{6}$ and theory would predict only 4.8 Raylelghs scattered solar light compared to $560 \mathrm{R}$ observed. Increasing $\tau$ would increase the scattered intensity very little and at $\tau=4$ the ratio of the opacity to the 1302 line $184 \times 10^{-4}$ which already seems rather high. Thus unless there is an undetected strong but perhaps narrow solar line at 1356 with peak intensity about 100 times the continuum intensity it would appear to be difficult to account for the 1356 line without assuming a local source of excitation.

\section{COMCLUSIONS}

This discussion should not be taken for a theory. It is an attempt, based on plausibility arguments, to 1solate the most important factors that determine the scattered intensity and to make an estimate of their contributions. The result should be reasonably close to what can be expected from a rigorous treatment besed on a wave-optical treatment and exact transport equations. The machinery exists for handing this problem adequately and it should be applied. Until then, however, this calculation will serve to demonstrate that the main features of the 1304 dayglow are 
possibly explicable in terms of resonance scattering of the wlar ur lines. The main uncertainty is in the absolute intensity. There is uncertainty as to what value to use for the effective solar flux at the time of the dayglow bservations and there is uncertainty as to whether the opread in reported dayglow intensities is real. This spread ranges from agreement wth the lower limit of Intensity expected for resonance scattering to almost four times that intensity. Whether there is a contribution from excitation by fast photo-electrons remains a question to be settled. Either It may be demonstrated that the excitation is as efficient as that required by Donahue and Fastie or it may be show that the dayglow peak at $180 \mathrm{~km}$ is too large to be accounted for by resonance scattering of sunlight. Neither has yet been conclusively done.

\section{ACKROWTEDGEYENMS}

This work was supported in part by the Kational Science Foundation, Atmospheric Sciences Section, and by the Fational Aeronautics and Space Administration under Contract MASAr-179. Very useful discussions are acknowledged with J. W. Chamberlain and T. Holstein. 
RIFLRBIICES

1. T. M. Donahue and W. G. Fastie, Space Research IV, Ed. P. Muller, Korth-Holland, Amsterdan, 1964.

2. T. A. Chubb, E. T. Byram, H. Frledman and J. E. Kupperian, Jr., Ann. Geophys. 14, 109, 1958.

3. W. G. Fastie, H. X. Crosswhite and D. F. Heath, J. Geophys. Res. 69, 4129, 1964.

4. W. G. Fastie and H. M. Crosswhite, Planet. Space Sc1. 2, 1021, 1964.

5. S. A. Kaplan, V. V. Katyushina, and V. G. Kurt, 5 th Internatienel Space Science Symposium and Cospar Neeting, Florence, Italy, vay, 1964.

6. R. Tousey, Space Sc1. Rev. 2, 3, 1964.

7. R. Tousey, J. D. Purcell, W. E. Austin, D. L. Garrett and K. G. Widing, Spece Research IV, Ed. P. Nuller, North-Holland, Amsterdam, 1964.

8. I. S. Borren, Pub. A. S. P. $46,146,1934$.

9. A. Dalgarno, Ann. Geophys. 20, 67, 1964.

10. C. Barth, Communication to Aeronomy Sympostum IUGG, Berkeley, 1963.

11. A. B. Prag and K. C. Clark, Phys. Rev. Letters 12, 34, 1964.

12. F. Kaufmann, J. Chem. Phys. 1965 (to be published).

13. R. H. Garstang, Proc. Cambridge Phil. Soc. 57, 115, 1961.

14. A. E. Hedin, C. P. Avery and C. D. Tschetter, J. Geophys. Res. $69,4637,1964$.

15. C. Reber, J. Geophys. Res. 69, 4681, 1964.

16. T. M. Donahue, J. E. Blamont and M. L. Lory, Planet. Space Sc1. 5, 3, 1961.

17. T. Holstein, Phys. Rev. T2, 1212, 1947. 
18. T. Holstein, Furs. Rev. 83, 1159, 1951.

19. I. M. Blberman, J. Expt1. Phys. U.S.S.R. 17, 416, 1947; 19, 584, 19 h.

20. I. Tohnatsu, to be published, 1965.

21. W. Unno, Pub. Astr. Soc. Japan 4, 100, 1952.

22. D. E. Osterbrook, Ap. J. 135, 195, 1962. 


\section{FIGURE CAPTIOIS}

Figure 1. Relative number of photons scattered from a continuum by a IIne with a pure Doppler shape and by a line with combined Doppler and resonance shape as a function of optical depth along the light path at the line center.

Figure 2. Relative rate of photon absorption per unit optical thickness along the primary beam in the Doppler core for a pure Doppler line shape $\left(\tau_{0} \sqrt{\pi \ln \tau_{0}}\right)^{-1}$ where $\tau_{0}=\tau / \mu$, In the wings $(\infty)$ and in the region of the wings from $x=2.85$ to $x=3.44, x=2.85$ to $x=3.55$ and $x=2.85$ to $x=4.26$. The altitudes at which various $\tau / \mu$ values are reached are indicated for $\mu=0.5$ in the 1306 line, the 1304 line and the 1302 line.

Figure 3. Total number of photons scattered from the primary beam in kilo Rayleighs for each line as a function of altitude. The observed altitude profile is shown as a dashed curve scaled by a factor of 20 .

Figure 4. Primary excitation rate of the ${ }^{3} \mathrm{~S}$ level relative to the rate for a pure Doppler line shape as a function of optical depth in the $1302 \AA$ line. The altitude in $\mathrm{km}$ is also marked.

Figure 5. First approximation to the emission rate and final emission rate multiplied by $\left(\pi F_{0} \sqrt{\pi} \Delta v_{D} / 0 \Delta v_{S}\right)^{-1}$ :

Figure 5. Predicted overhead intensity in kllo-Rayleighs as a function of altitude (solid line). Observed profiles normalized at $185 \mathrm{~km}$. Dots are observations by Fastie, Heath and Crosswhite in 1963, circles same group in 1962 and dashed curve the results obtained by Kaplan, Katyoshina and Kurt for a solar zenith angle of $87^{\circ}$.

Figure 6. Observed intensity of the $1356 \AA$ line in Ravleighs. Theoretical profiles are for two different opacties beginning at $118 \mathrm{~km}$ and $122 . \mathrm{mm}$. 


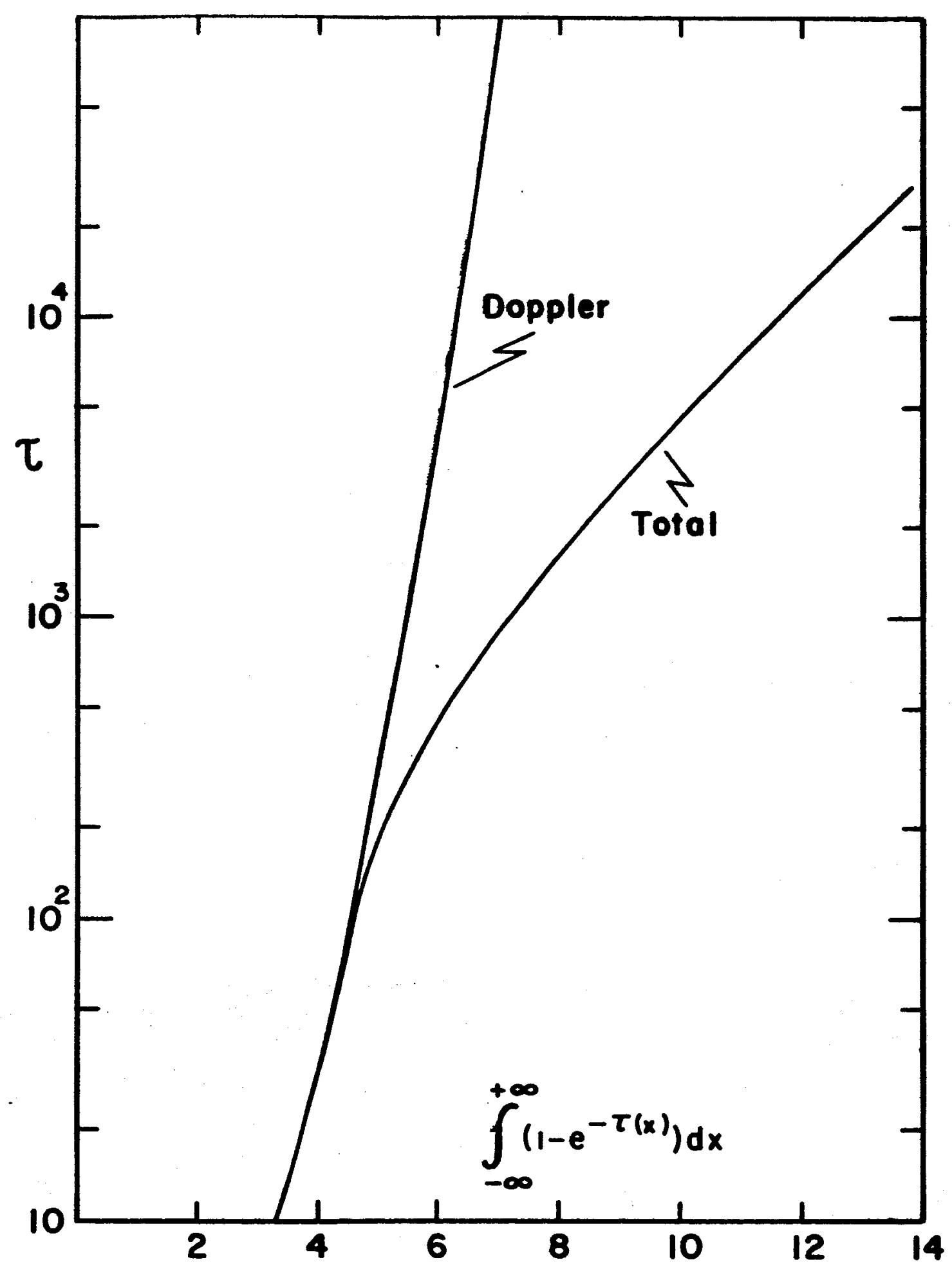

Fio. I 


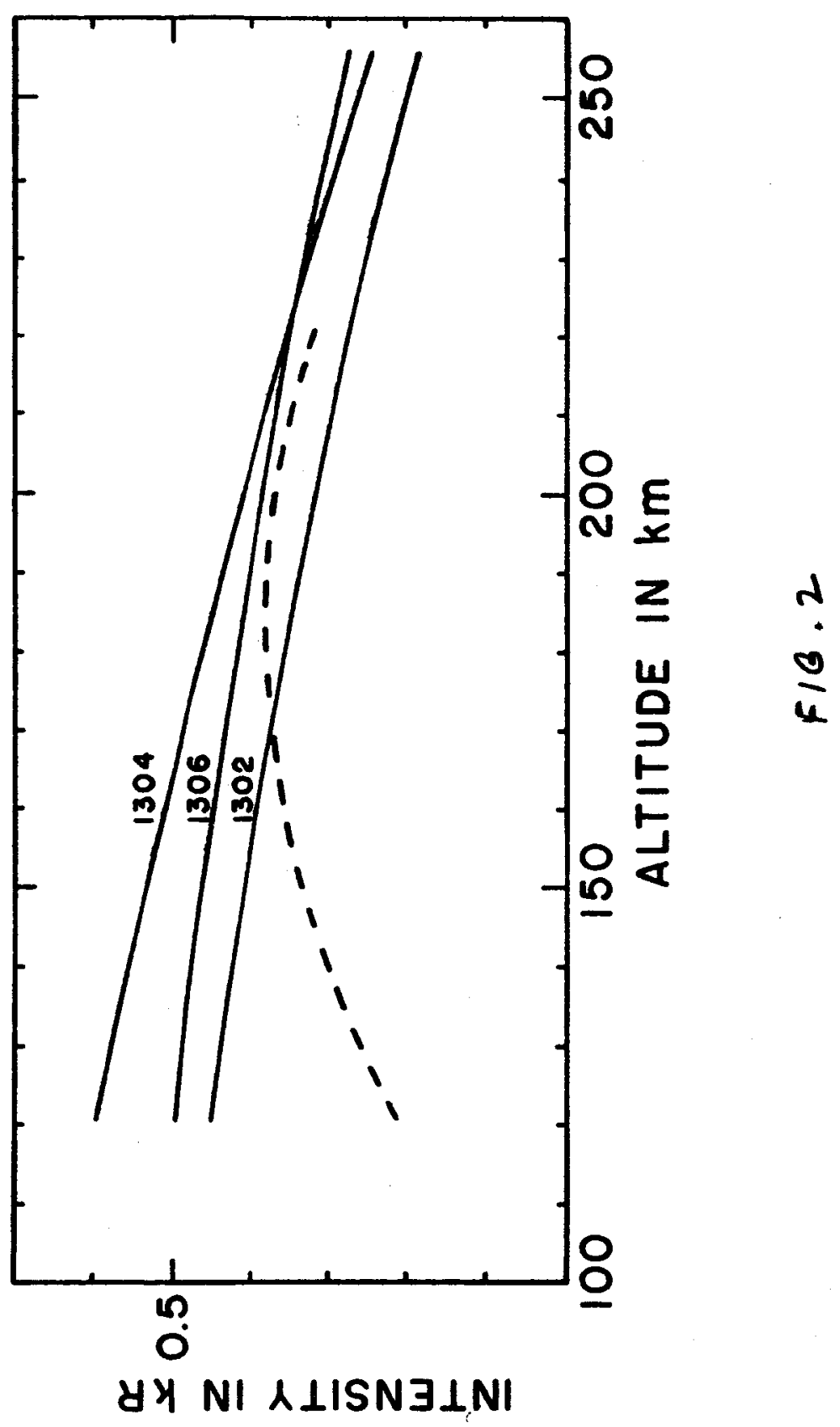




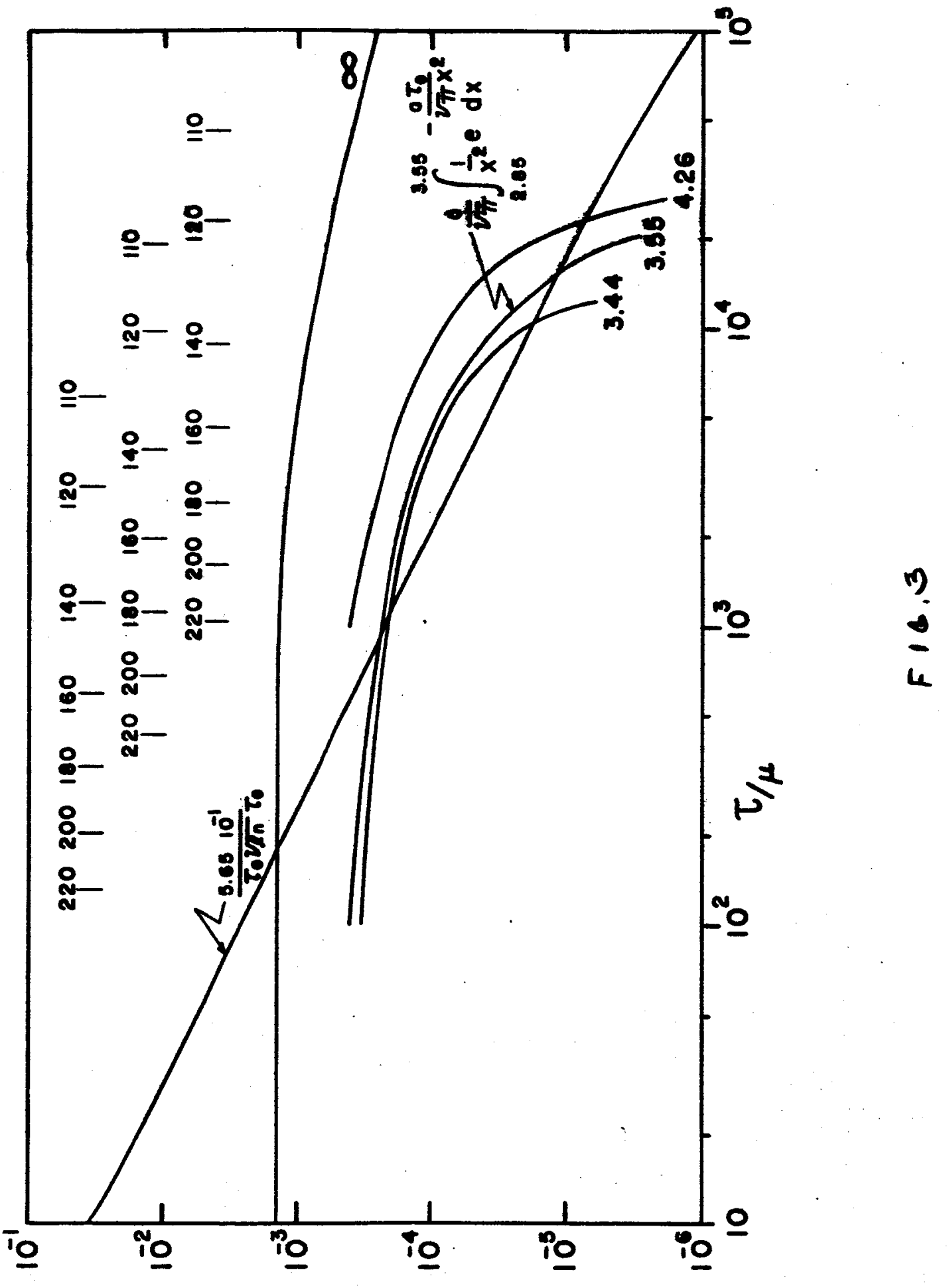




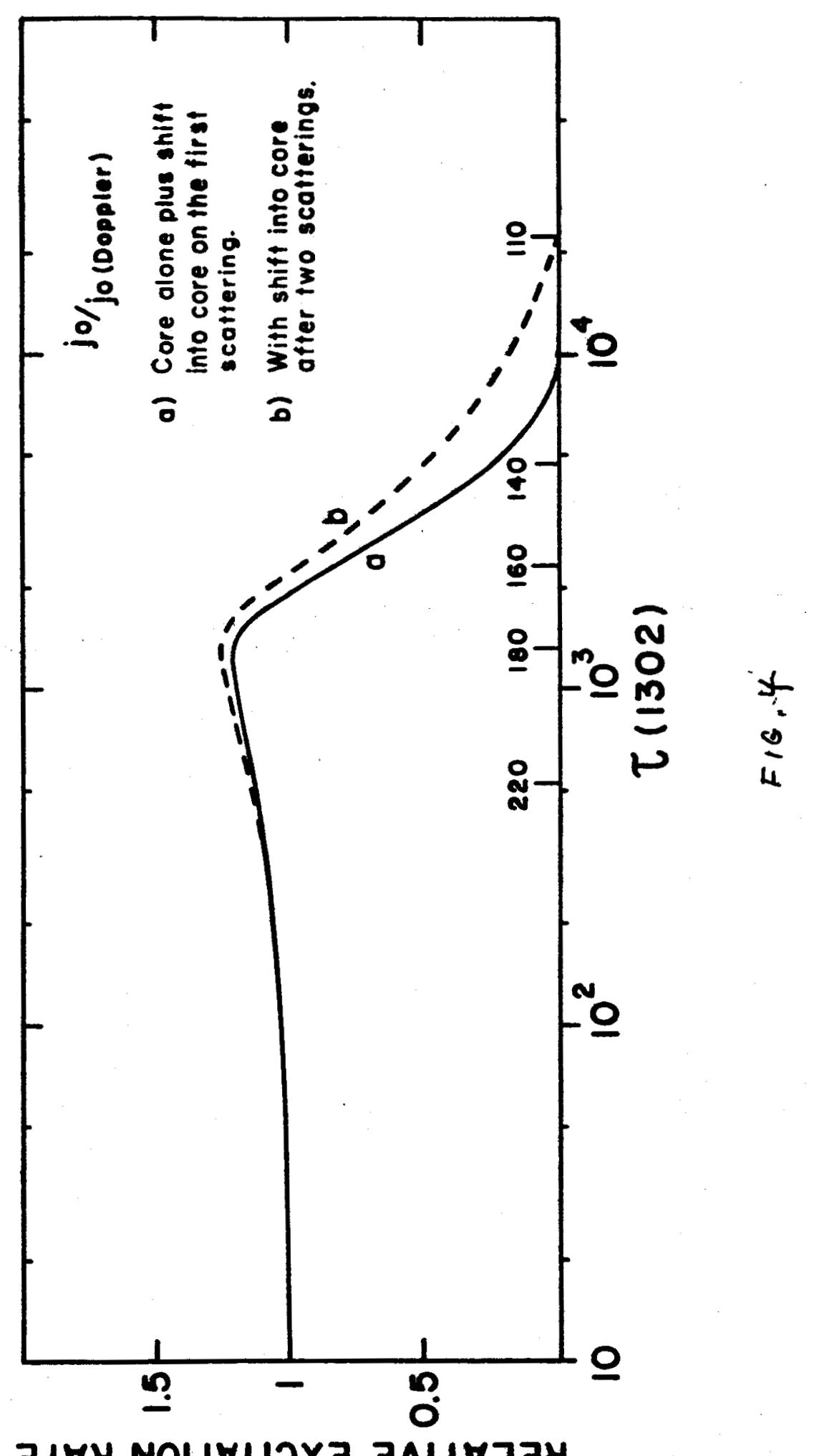




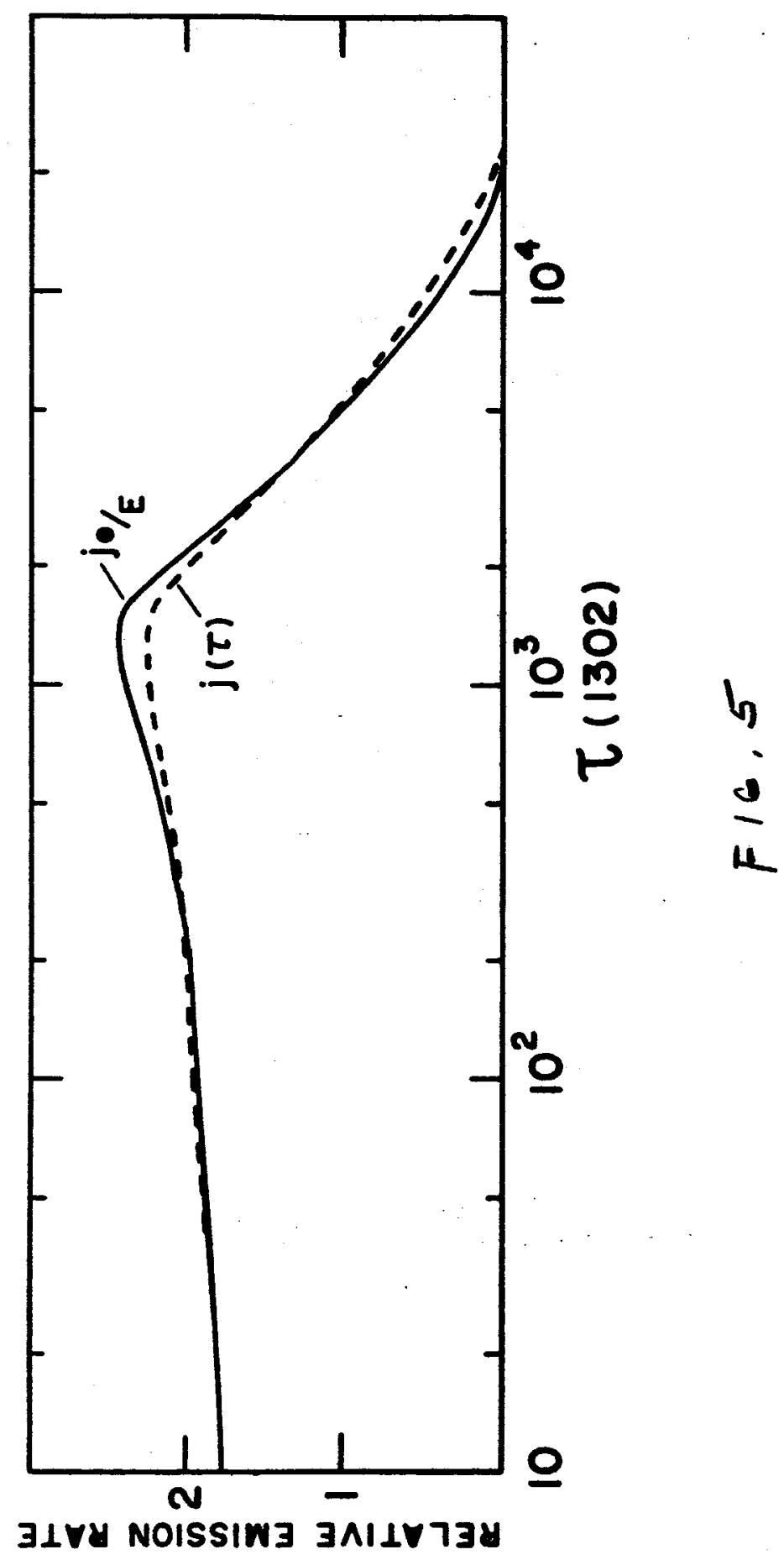




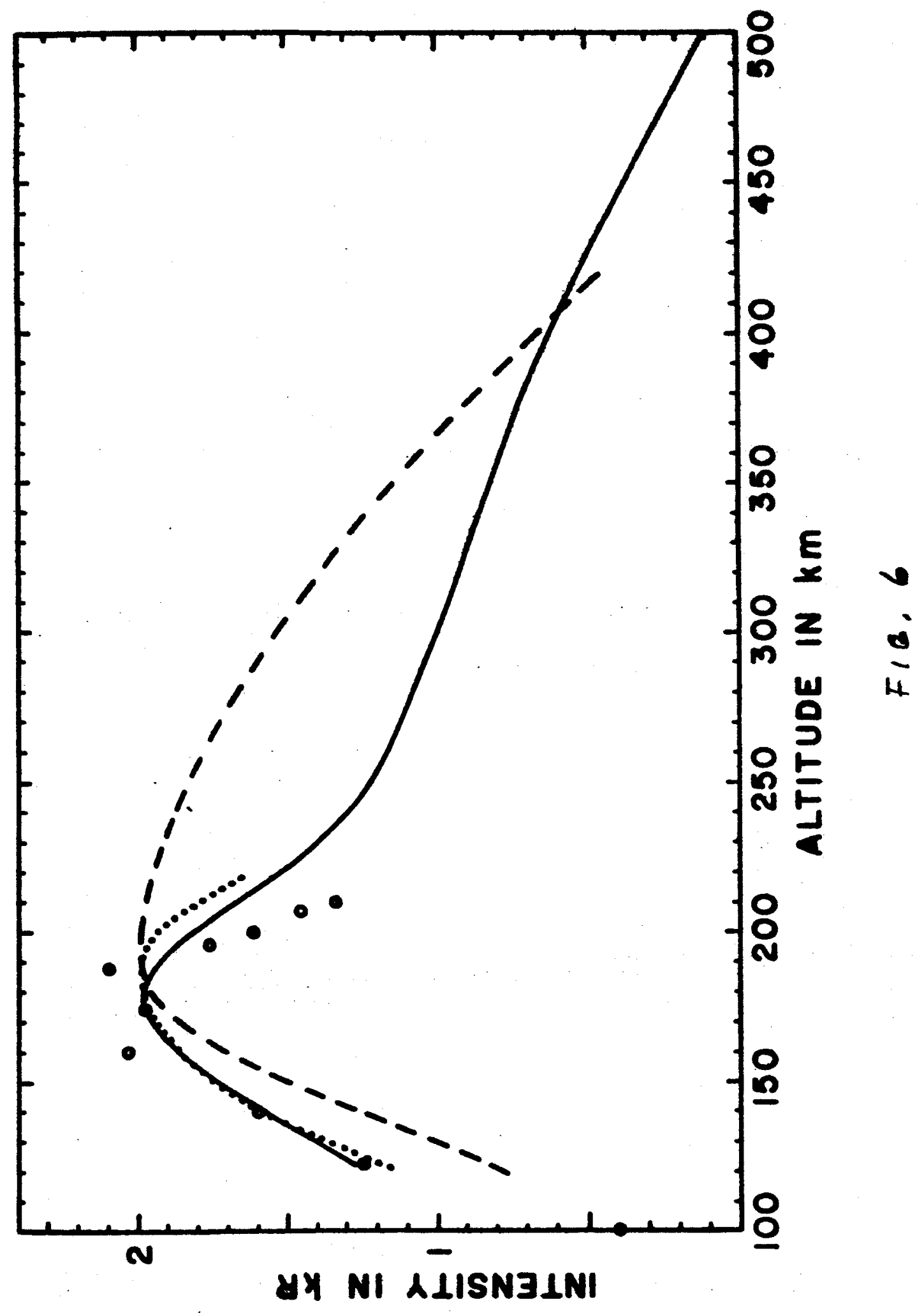




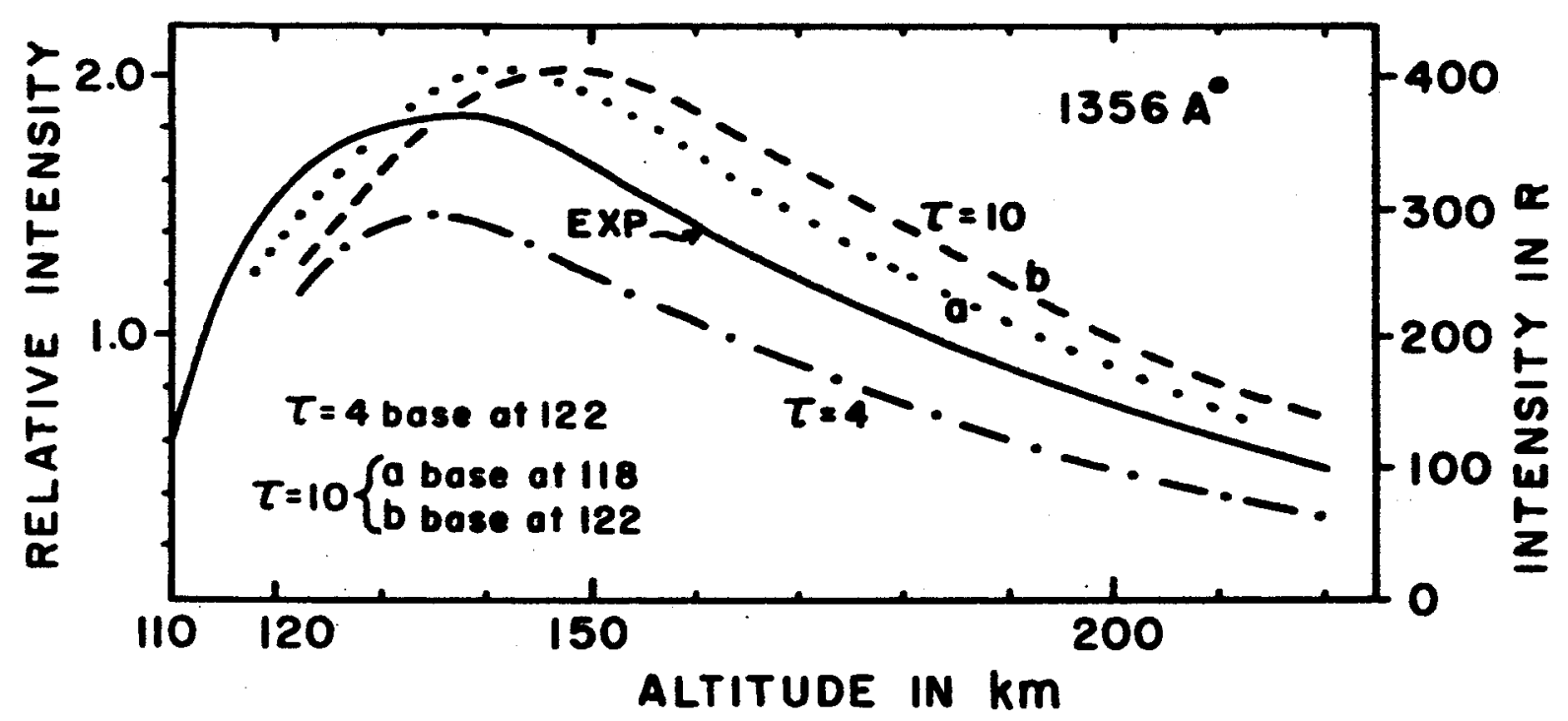

F,G. 7 